\title{
Meeting the Needs of Children and Families with Communication and other Developmental Difficulties in Accra, Ghana-An Assessment of the AwaaWaa2 Community-based Program
}

\author{
Tricia Stephens $^{1}$ - Nana Akua Victoria Owusu ${ }^{2} \cdot$ Tyrone Parchment $^{1} \cdot$ Mary McKay $^{1}$
}

Published online: 28 April 2015

(C) Springer International Publishing 2015

\begin{abstract}
The families of children living with communication and developmental difficulties in Ghana face challenges in accessing quality care. In the absence of an organized governmental response to the needs presented by these children, a handful of grassroots providers has formed what may be described as an emerging special needs child serving system. This qualitative study looks at the lived experiences of nine families raising children with a range of communication and other developmental difficulties. In-depth interviews were conducted through a collaborative effort between AwaaWaa2, a local community-based organization providing provisional services to children, and the McSilver Institute for Poverty Policy and Research. Results indicate that families are finding innovative ways of meeting the needs of their children. A comparison with an assessment conducted in 2006 was done. Partnerships like the one forged between these two organizations are a potential tool in awareness raising and advocacy. Implications for global and grassroots community level collaboration are discussed.
\end{abstract}

Keywords Child communication difficulties $\cdot$ Child developmental difficulties $\cdot$ Community collaboration . Ghana $\cdot$ Global social work $\cdot$ Advocacy

Tricia Stephens

tns205@nyu.edu

1 McSilver Institute for Poverty Policy and Research and NYU Silver School of Social Work, 41 East 11th Street, New York, NY 10003, USA

2 School of Allied Health Sciences, University of Ghana, Legon, Ghana

\section{Introduction}

The ability to communicate clearly is a highly prized skill and passport to success in most cultures. Communication forms the cornerstone for social, emotional, and cognitive well-being. Though the ability to communicate verbally is central to childhood development, problems with communication are often not detected early on (Landa et al. 2007; Siklos and Kerns 2007). While advances have been made in early detection, communication difficulties along with other early developmental delays can be difficult to identify accurately (Cox et al. 1999; Wetherby et al. 2007). These are issues faced globally but which are better managed in better-resourced countries. In countries like Ghana, with competing requirements for health-care dollars and facilities, the task of providing services to children with communication and other developmental disabilities is daunting.

Whether delays in recognition of communication and other developmental difficulties (CDD) are a result of normalizing a child's progress as being on their own developmental trajectory or misdiagnosed by health-care providers, most CDDs become apparent around the 24-month mark or upon the advent of school entry (Baron-Cohen et al. 1996; Scherzer et al. 2012). The burden of late detection and an absence of treatment options for these children affect all areas of functioning (Olusanya and Newton 2007). This is even more so in cultures, like Ghana, where oral and face-to-face communication seems to be the preferred means of communication dominating everyday life (Crowley et al. 2013).

The most prevalent, obvious, and better-documented disabilities in Ghana are those that are visual, auditory, and physical (Avoke 2002; Tuakli-Wosornu and Haig 2014). These disabilities receive a disproportionate amount of attention and funding in a country with demands that vastly outpace available resources. As noted by Olusanya et al. (2006), where 
health services are available in the developing world, they are usually geared toward more obvious disabilities and/or betterfunded or global priority illnesses. This pulls resources away from the community of children in Ghana who live with CDD. Delays in recognizing and treating CDD can have profound effects on the way in which a child is then introduced and integrated into the larger society. The socioeconomic implications of the current gap in service provision are alarming as a significant proportion of the population is denied what should be basic human rights: the right to effective oral communication and the opportunity to compete and survive in a challenging and communication-driven world.

Individuals with disabilities have higher rates of undereducation (Tuakli-Wosornu and Haig 2014). The delivery of disability-specific services and the preparation of special education teachers in Ghana are limited to disabilities relating to vision, hearing, and mental retardation. This caters to a very small number of the children with special education needs. The focus on this subgroup of children is understandable given that they are some of the most disadvantaged and marginalized (Crowley et al. 2013). It does however leave the greater number of children with CDD without much needed attention even though they are equally prone to marginalization and exclusion. Basic universal early educational services are nascent with anywhere between 27 and $42 \%$ of Ghanaian children aged 3 to 5 years having access to early childhood education (UNESCO 2006). This means that many children with CDD stand very little chance of receiving any education during their early years. Steps are needed to interrupt the trajectory many of these children end up on as a result of limited access to appropriate care (Adera and Asimeng-Boahene 2011).

Stigma, ranging in intensity from shunning/avoidance to placing spiritual blame on the parent (primarily the mother) for what is happening with their child, remains a very real challenge for children and families living with CDD in Ghana. Parents must navigate a virtual minefield when seeking services for their children (Reynolds 2010). The relationship between community resources and attitudes toward the disabled indicates that better-resourced communities tend to be more accepting and supportive (Munyi 2012). This contextual lens, along with gains in education and treatment, may have far-reaching impact in combating stigma in Ghana where reliable information about the disabilities and treatment options is limited.

\section{Prevalence}

Precise numbers on the prevalence of childhood CDD are very difficult to come by in developing nations due to insufficient diagnostic services and inconsistent record keeping. Reasonable attempts at triangulation of prevalence can be made through larger population estimates. According to the
United Nations, approximately $15 \%$ of people in any given population may have a disability including a communication disorder (United Nations Convention on the Rights of Persons with Disabilities 2006). According to the 2010 Ghana census, there are approximately 25 million people living in the country (Ghana Statistical Services 2012) thus indicating that there may be up to 3.75 million Ghanaians living with a disability. Ghana has a youthful population with $38.3 \%$ of the population or 9.5 million people recorded as being aged 15 years and under (Ghana Statistical Service 2012). Using the UN estimates, it is possible that there are up to 1.4 million children aged 15 and under with some form of disability in Ghana.

Additional studies have been done that give statistics specific to Ghana's school-aged children. Approximately 14.4 out of 1000 children ages 1 to 5 years living in Ghana has a disability according to a 2001 study conducted by Biritwum et al. (as cited in Tuakli-Wosornu and Haig 2014). Children within that population base are calculated at one in eight of all preschool children with some form of disability at some point in their early years' experience (Anthony 2011), with an increase in prevalence anticipated with an increase in age (Tuakli-Wosornu and Haig 2014). These numbers indicate the number of children potentially struggling with CDD could be in the thousands. The scope of the need is profound given the relative dearth of services available.

\section{AwaaWaa2}

AwaaWaa2 is a community-based educational center catering primarily to the needs of children with communication difficulties (CD). It was established in 2006 following a study of the availability and accessibility of services for children with communication difficulties in Ghana conducted by the center's founder Nana Akua Owusu. AwaaWaa2 is one of only a handful of providers in Accra, Ghana, that have been established by private citizens to address the unmet needs of children who display signs of interruptions in what is considered normal development. Owusu, a speech and language therapist (SLT) and coauthor of this study, embarked on a needs assessment in 2006 to investigate and establish the existing level of need for children with communication difficulties. The information obtained through that study was to be used to inform service provision to children, families, and providers of CD services both in Ghana and with large Ghanaian communities in the UK. Recognizing the significant need and the inaccessibility of services and trained providers, Owusu repatriated to Accra, Ghana, and founded AwaaWaa2, which is staffed by a small team of teachers, parents, and volunteers. While AwaaWaa2 was originally established to meet the needs of children with communication disorders, which according to Olusanya et al. (2006) includes speech, language and hearing disorders, it soon became a beacon for children with a range of developmental difficulties including 
confirmed and suspected incidents of autism and cerebral palsy among others.

\section{Versus 2014 Needs Assessments}

The findings of the 2006 needs assessment confirmed an alarming gap in the provision and delivery of services for children with $\mathrm{CDD}$ and their families. A common problem for many parents who took part in the initial study was that their early concerns about their child were dismissed. Some parents attributed this experience to a clear lack of understanding of critical language skill development and treatments on the part of some health-care workers. Parents also noted that teachers had often attributed their child's poor behavior to be a consequence of their inadequate parenting styles and not to their communication disability. In 2006, this constituted a delay in recognizing CDs in children in Ghana further complicating an already challenging disability.

This 2014 assessment compares the experiences of children and families both with communication and other developmental difficulties 8 years later to outline any changes in the timeliness of detection or treatment of CDD.

The following research questions were asked:

1) What is the process for families of children with CDD in accessing services in Accra, Ghana?

2) How do they view the services offered at AwaaWaa2?

3) Is there integration into the larger traditional school system for children with CDD?

4) What are the outstanding identified needs of these children and families?

\section{Collaborative Global Social Work}

A range of descriptions and definitions of participatory or community collaborative research has been offered (Baum et al. 2006; Creswell 2003; Strauss and Corbin 1990). There is agreement on some central themes and core foundational principles of such research efforts. It is generally accepted that participatory action or community collaborative projects are undertaken with the specific intention of creating change. Community stakeholders and researchers work together closely to undertake a reflective process addressing the current circumstances facing stakeholders and how they can be changed. Further, what distinguishes community collaborative efforts from other research approaches is the emphasis on the intensive and ongoing participation and influence of community members in creating knowledge (Israel et al. 1998). This theoretical framework informed the way in which all the key stakeholders approached participation in the study.

Research questions that result from strong collaboration between researchers and community members are crafted in partnership and tend to reflect the pressing concerns of community members and acknowledge the importance of indigenous knowledge and resources rather than over-relying on expertise brought by the university/institute partner. In sum, community collaborative research activities are defined by: "(1) a recognition that community development is an important focus on research activities, (2) a commitment to build upon the strengths and resources of individual communities, (3) ongoing attention to involvement of all members of the collaborative partnership across phases of a research project, (4) an integration of knowledge and action for mutual benefit of all partners, (5) the promotion of a process that actively addresses social inequalities, (6) opportunities for feedback, (7) addressing health from both strength and ecological perspectives, and (8) disseminating findings and knowledge gained to all partners" (Israel et al. 1998; p. 178-179).

This study is an example of moderate-intensity global collaboration between a research institute and a community partner, which is in its early stages. AwaaWaa2 is established in the Ghanaian community of children and families with CDD and is also well known among health-care providers. There was strong buy-in of this research project from the study staff and parents who were excited to partner with a highereducation research institute that might provide additional resources and insight into how to best help their children. The research agenda and the decision-making power were shared between the researchers and the community partners. Initial contact between the university team and the community-based organization was made through a mutually known human rights organization based in Cape Coast, Ghana. Once the introduction was made direct communication between the university partners and AwaaWaa2 ensued with planning for a week-long intensive study on site during the summer of 2014.

The university partner created a team that consisted of social work and public health academics, practitioners, administrators, and master's level social work students. Students were enrolled in a course on human rights and mental health in Ghana and received instruction both in the USA and in the social work department at a major university in Accra. Research content on conducting needs assessments and interviews was covered in the classroom with an opportunity for practice interviews prior to arriving at AwaaWaa2. As noted by researchers Parker et al. (2014), it is essential to create an atmosphere of bi-directional learning in global social work settings, and this core concept was carried forward throughout the course and the subsequent analysis of the data.

\section{Methods}

A total of nine interviews with parents/caretakers were conducted using a semi-structured interview guide. The staff at AwaaWaa2 vetted the semi-structured interview for 
appropriateness and relevance to the parents prior to its use. Given the difference in culture, particular attention was paid to phrases or language that were not relevant in the Ghanaian context. MSW student interns worked in pairs with one student serving as the interviewer and the other as a note-taker. Interviews ranged from about an hour to over $2 \mathrm{~h}$ in length and were audio recorded. Recordings were then transcribed with two interns participating in initial review and preliminary theme development. These preliminary themes were shared with the staff and administrators prior to the university team's departure from Accra.

\section{Data Analysis}

Nine parent/caregiver transcripts were analyzed using a multiphase, iterative process that included the use of the qualitative data analysis software Dedoose. All transcriptions were uploaded to Dedoose thus allowing for collaborative coding and analysis. Two doctoral social work candidates worked together to conduct the data analysis. One of the candidates was also present during data collection in Ghana and thereby had rich contextual knowledge of the interviews.

Each transcript was read and analyzed using a narrative analysis approach (Padgett 2008). Padgett noted that data analysis in narrative approaches to data collection needs to capture meaningful information that is strewn throughout the natural flow of discussion in an interview. The flow of the interviews lent themselves to this form of analysis, while the interview guide that was developed prior to data collection was helpful in organizing the data. Each coder independently developed a set of codes for the transcripts. This open-coding phase of the data analysis included assigning themes to sections of the data ranging from phrases to several paragraphs (Burnard 1991). Once open-coding was completed additional themes were developed to describe relationships between broader categories and subcategories, a process known as axial coding (Strauss and Corbin 1990). The use of this approach in axial coding allows for a more systematic and organized secondary coding of the data. Both coders then compared the codes they had developed, retaining codes that overlapped, discussing divergent codes to produce a final version of the codebook.

The use of the data analysis software Dedoose was helpful particularly in the area of measuring inter-rater reliability. Using the test section of the software, the two coders compared randomly selected excerpts. The initial inter-rater reliability was 0.65 , unsatisfactory for this study. In discussing this large gap in scoring, the raters deduced that it was the hands-on experience of one rater that frequently shifted her rating of particular codes. Thorough discussion and subsequent randomly selected coding of 20 excerpts over three attempts resulted in an inter-rater reliability coding of 0.85 .

\section{Results}

\section{Participants}

Nine caregivers inclusive of seven biological parents and two extended family members were interviewed using the semistructured interview guide. These nine caregivers accounted for ten children (one family had two children enrolled). The community that accesses services through AwaaWaa 2 is small and close-knit. For this reason, no identifying demographic information was collected nor will any be used in this study. Instead, respondents will be referred to as mother or father or caregiver. This affords a greater degree of confidentiality in reporting the findings.

\section{Study Questions}

(1) What is the process for families of children with CDD in accessing services in Accra, Ghana?

A delay in speech was most frequently cited as the primary reason that parents sought help for their children. There were parents who described having a feeling that their child was not progressing along a normal trajectory, but these parents stated that it was the delay in speech that motivated them to seek help. Parents described initiating their help-seeking starting when their child was as young as 12 months old and extending to 24 months and older. In addition to speech delays parents described: child social problems including poor relatedness to others, and child not engaging in play with others; child developmental delays including delayed speech and inability to feed oneself; communication delays including child not acknowledging their name and not responding to direct communication requests from others; and some behavioral issues including difficulty sitting still for more than a minute at a time, screaming, or throwing tantrums.

One father described his son's current level of functioning,

When we take him to church, all other kids are around him. They like him so much. But he doesn't play with them. He will be doing his own thing.

One mother said,

... It was actually someone who mentioned that. She asked me if my son had said mama yet. I said no. He was only two so I wasn't expecting much, but after awhile I realized it was really, really bad. Even simple things, you tell him to come and he didn't even realize his name. I realized it was a problem and we realized it was a problem and we went to see a specialist and he referred us here. 
The primary care physician was the first point of contact for most parents who sought help for their child. The route of referral was frequently from a family physician/pediatrician either directly to a speech therapist that recommended AwaaWaa2 or to a neuropediatrician who then made the referral to the center. The hospital-based neuropediatrician at Korle $\mathrm{Bu}$ Hospital (Ghana's main teaching hospital, located in Accra) referred parents to the speech therapist who in turn made referrals to AwaaWaa2. Two parents had repatriated to Ghana from Western countries and had had some preliminary evaluations or had been advised to have evaluations done in the country of the child's birth.

A mother who had resided her entire life in the country and who had two children enrolled stated,

...It started when he was around two that is when we were concerned... [W] hen first went to see a pediatrician he said that the boys were like that so we should give it some time so we waited so we didn't go to Korle Bu when he was 2 . We waited for maybe about 6 months and there was no improvement [We] went back and he still said we should wait and that is when we said ok let's go ahead and do the hearing tests.

Families repeatedly expressed their dismay at the severe lack of services available to their children and stated that these problems were systemic. Noting limited health-care resources, receiving inaccurate information and blaming responses from people outside of and sometimes within their family system, parents were often simultaneously overwhelmed and relieved by the time they accessed services at the center.

A mother who had repatriated to Ghana described her impressions of the classroom setting at AwaaWaa2,

...But I am expecting the very best for my son. I am expecting highly qualified people who exactly know what they are doing. I am not sure of exactly what training they have, but I can see that his teacher knows what she is doing. The others seem to be helping. I would wish that everybody in the team has some knowledge of how to care for these kids and how to engage them. I feel it's a lot of work for her. If maybe there were three or two, it would be easier for her, because they [the children] have very short attention spans. One starts running, they won't listen. That's why all the parents we come around keep our kids calm. But this is the best we have right now. So we take whatever we can get

Yet another mother reported,

Oh! It's terrible. Very very terrible. So difficult, you don't even know where to look. It's just this place and two or three places that came up. Same thing over and over again,..., they are so expensive. Extremely expensive.

(2) How do they view the services offered at AwaaWaa2?

Parents were mostly pleased with the level of care that their children received at AwaaWaa2. While they noted that they had very limited choices and that the expense of enrollment was significant for their family, they felt that AwaaWaa 2 was one of a handful of places in the country where their children could receive help. Many parents were very involved in their child's care and would sit in while they were in school or arrange for a family member to remain at the center with their child all day. Most families had extended family support and or caretakers that lived in the home and shared caretaking responsibilities for the children.

The length of enrollment varied greatly. Some parents had been at the center for less than 3 months while others had their child enrolled since the inception of the center. The extended length of time some children remained enrolled was emblematic of the lack of services available to parents - simply stated parents had nowhere else to take their child. Parents described satisfaction with the level of warmth and attentiveness of the teachers. They also expressed joy and hope in the achievements they had noticed in their child since their child began attending AwaaWaa2. Parents described significant gains in their child's receptive knowledge, ability to follow instructions, use of gestures, and, for some, in their child's level of relatedness. They described their joy in progress such as their child being able to hold eye contact, eat independently, or sit and engage with other family members.

One mother explained,

Yea. She used to keep to herself. Now she mixes with other kids and the people here.

Yet another mother stated,

They are helping her to come up. It's a challenge. Little by little... I have a poster of the body and I put it on the wall in the house and she was not paying attention [before], but now if I see her there, I just move in and start pointing. I will just hold her hand and she will be saying one, two... It's a slow process, but by the Grace of God, through this interaction and by the Grace of God, she will be improving and giving me courage.

Parents were able to access support from other parents when they went to AwaaWaa2. Many parents, recounting social isolation and in some cases ostracism, expressed relief at finding a community within AwaaWaa2. The informal support provided by other parents and staff and volunteers who have 
children with CDD was cited as being a strength and resource to caregivers.

As another mother put it,

... Yes. I learnt it from here [AwaaWaa2]. I learnt quite a lot. Encouragement as well. Those were the years when I hid her in my home and didn't want others to know my child was like that. It was here that I was able to pour it out. I come and I talk. ....At least [now] my child is able to indicate what she wants and it helped me because it got to a time, before I started talking, between the age of 1 and- 3, I think I was dying myself. My blood pressure went up, so frustrated, angry at almost everything and everybody. The frustration was so severe. But then I met parents at Awaawaa2. They told [me] relax and cool down. You are not alone in this. The other people have gone through the same thing and give me the courage. It is the hope that gives me strength.

(3) Is there integration into the larger traditional school system for children with CDD?

Most parents reported that their child was integrated into the mainstream school system on some level. Attendance at the mainstream school ranged from 1 to 4 days out of every week. An AwaaWaa2 staff person or volunteer accompanied the child to school to assist them in their daily tasks. The plan for most children was to increase their days in the mainstream school while attending AwaaWaa2 to work on building attention and getting accustomed to sitting in a classroom. Of the parents and caregivers interviewed, only two indicated that their child attended AwaaWaa2 only. The stated goal for each caregiver interviewed was that their child would be able to be fully integrated into the mainstream school as quickly as possible. For this reason, parents emphasized a desire that their child display skills like "be able to hold a pencil" and "I want him to speak, yes he must speak." Some parents expressed a desire that AwaaWaa2 be converted into a "regular school for the children" where their children could learn a curriculum that more favored the traditional school setting.

(4) What are the outstanding identified needs of these children and families?

Caregivers were clear that their families exist within a gap in service provision in Ghana. They described nearly nonexistent specialized health-care follow-up for their children, nonexistent public education facilities, and ongoing stigma directed toward them and their child in the broader society. For those families with access to resources outside of the country, they actively sought out specialized trainings, utilized access to resources made available through family members living overseas, and utilized internet resources where available. All of these efforts were made in the absence of organized services for their children.

Some parents had taken on the mantle of educator and advocate. They detailed their outreach efforts within their communities to other parents who have continued to keep their child with CDD indoors. They described their efforts to include their child in their social activities in spite of stigmatizing responses from some community members. Parents and caregivers also shared their thoughts on how their children could be more fully integrated into youth life by providing education programs in schools and organizing events where their children have opportunities to be around the mainstream students in social settings. It is important to note that stigma remains a very real part of the experience of both children and families with CDD in Ghana. One parent noted that once her child was integrated into the mainstream school, the teacher for that classroom resigned citing fears of contagion from their child. Persistent beliefs concerning spiritual blame for disability present a challenge to those who would like to realize gains in inclusion and integration.

Caregivers had specific ideas for how to improve the services that were available to their children at AwaaWaa2. They recognized that only some of the center staff appeared to be trained and so requested both an increase in the number of teachers available on site and trainings be available to those teachers. Those parents who had identified speech delay wanted more time with the speech therapist who is currently on site 1 day a week.

A father stated:

I want you to increase the time where the contact is with the speech therapist. You know it is only Tuesday with the children with the speech issues.

They noted that additional recreational material that promoted play and expression through music be incorporated into the resources available at AwaaWaa2. Most of the caregivers highlighted their child's receptiveness and responsiveness to music and asked for more opportunities for them to express themselves creatively.

... improvement. Something like, if we have something they can be using. My daughter likes to be singing. She knows the song, any song.

\section{Limitations}

The small sample size and nonexperimental method employed in this study present a major barrier to generalizing the results obtained. A longitudinal perspective, including revisiting some of the parents and health-care and educational providers initially interviewed in 2006, would have strengthened the 
methodology. Nonetheless, the information gathered in this study remains a useful tool in explicating the phenomenological experiences of parents with children with CDD in contemporary Ghana.

\section{Discussion and Implications}

Services for children with CDD are minimal in Ghana. The lack of an organized governmental response to service provision for children with communication and other developmental difficulties has not changed significantly since the 2006 study. At the conclusion of the initial needs assessment, a recommendation was made that a comprehensive diagnostic and treatment center be established where families could receive wrap-around services including early and accurate diagnosis and treatment follow-up. The experiences described by these parents indicate that outside of the grassroots efforts in the private sector, service provision remains absent at the public level and thus remains inaccessible to the majority of children and families living with CDD in the country.

Organizations such as AwaaWaa2 were created specifically for children with communication difficulties and receive referrals from all corners of the country. In spite of the intention to solely treat those with communication difficulties, the lack of services has resulted in the center providing care to children with other developmental difficulties. In the absence of an organized governmental response to the needs present in the country, parents and a handful of professional providers have organized at the grassroots level to pull together services that meet their children's needs. At times, using information garnered through the use of technology or where possible traveling to get information relevant to their child, these parents have shown great tenacity and determination in the face of overwhelming circumstances.

Families of children with CDD continue to face significant stigma in their communities. The tendency to isolate themselves and their children can be seen as a coping mechanism to avoid interactions where they are blamed or where their children receive disapproving comments or stares. Parents indicated a need not only for access to reliable information but also for those in the broader society, including schools, to be educated on the facts about CDD. They believe that this could potentially reduce some of the stigma that they face in their day-to-day interactions. Calls for broader integration of children with CDD into the educational and social lives of their peers are also a long-term strategy to build alliances for those who suffer with disabilities in the long term. The hope is that those children will grow up to have a more inclusive outlook and serve as potential allies for the disabled in Ghana.

While providing a haven for children and their families with CDD, AwaaWaa2 is only beginning to put a dent in the needs that these families have. Reflective of the broader needs of the nation, staffing and training gaps stifle the ability of those on the ground to provide the quality of service they would like to. In a study of community-based organizations following the termination of international financial support, Kuyini et al. (2011) recognized the challenges that these organizations faced in the absence of external funding. Some of the challenges include a heavy reliance on volunteer labor and diminished support from governmental entities. Future studies on whether 2012 legislation mandating equal access to education for all children in the country will have an impact on funding and support are needed. Parents and providers continue to labor at the grassroots level to make this legislation a reality for their children with CDD.

Collaboration with larger global institutions like the one that took place in the summer of 2014 can make it possible to conduct the kind of research that produces knowledge that can be used for advocacy and awareness raising. The results of the needs assessment are shared knowledge between AwaaWaa2 and the research institute in accordance with the guidelines of best practices for community collaborative work. This allows each organization to use the information as a platform for effecting change in the area of supporting children and families who cope with CDD in Ghana. The results are slated to be used in talks with government representatives to forefront the needs of these children and lobby for the allocation of funds to develop services that meet their needs.

Acknowledgments Funding for this project was secured through an internal grant from the McSilver Institute for Poverty Policy and Research.

Many thanks to the parents and caregivers of the children with communication and other developmental difficulties who agreed to participate in this project.

Special thanks to the staff and volunteers at AwaaWaa2 who shared their perspectives, time and thoughts with the students and faculty from the Silver School of Social Work at New York University and the McSilver Institute for Poverty Policy and Research.

\section{References}

Adera, B. A., \& Asimeng-Boahene, L. (2011). The perils and promises of inclusive education in Ghana. The Journal of the International Association of Special Education, 12(1), 28-32.

Anthony, J. (2011). Conceptualising disability in Ghana: implications for EFA and inclusive education. International Journal of Inclusive Education, 15(10), 1073-1086.

Avoke, M. (2002). Models of disability in the labelling and attitudinal discourse in Ghana. Disability \& Society, 17(7), 769-777.

Baron-Cohen, S., Cox, A., Baird, G., Swettenham, J., Nightingale, N. A. T. A. S. H. A., Morgan, K. A. T. E., \& Charman, T. (1996). Psychological markers in the detection of autism in infancy in a large population. The British Journal of Psychiatry, 168(2), 158-163.

Baum, F., MacDougall, C., \& Smith, D. (2006). Participatory action research. Journal of Epidemiology and Community Health, 60(10), 854. 
Biritwum, R. B., Devres, J. P., Ofosu-Amaah, S., Marfo, C., \& Essah, E. R. (2001). Prevalence of children with disabilities in central region, Ghana. West African Journal of Medicine, 20(3), 249-255.

Burnard, P. (1991). A method of analyzing interview transcripts in qualitative research. Nurse Education Today, 11(6), 461-466.

Cox, A., Klein, K., Charman, T., Baird, G., Baron-Cohen, S., Swettenham, J., \& Wheelwright, S. (1999). Autism spectrum disorders at 20 and 42 months of age: stability of clinical and ADI-R diagnosis. Journal of Child Psychology and Psychiatry, 40(5), 719-732.

Creswell, J. W. (2003). Research design: qualitative, quantitative and mixed methods approaches (2nd ed.). England: Sage Publications. London.

Crowley, C., Baigorri, M., Ntim, C., Bukari, B., Oseibagyina, A., Kitcher, E., \& Laing, A. (2013). Collaborations to address barriers for people with communication disabilities in Ghana: considering the world report on disability. International Journal of Speech-Language Pathology, 15(1), 53-57.

Ghana Statistical Service (2012). 2010 population and housing census: summary report of final results. Retrieved from: http://www. statsghana.gov.gh/docfiles/2010phc/Census2010_Summary_ report of final results.pdf on October 20, 2014.

Israel, B. A., Schulz, A. J., Parker, E. A., \& Becker, A. B. (1998). Review of community based research: assessing partnership approaches to improve public health. Annual Review of Public Health, 19(1), 173-202.

Kuyini, A. B., Alhassan, A. R. K., \& Mahama, F. K. (2011). The Ghana community based rehabilitation program for people with disabilities: what happened at the end of donor support? Journal of Social Work in Disability \& Rehabilitation, 10(4), 247-267.

Landa, R. J., Holman, K. C., \& Garrett-Mayer, E. (2007). Social and communication development in toddlers with early and later diagnosis of autism spectrum disorders. Archives of General Psychiatry, 64(7), 853-864.

Munyi, C. W. (2012). Past and present perceptions towards disability: a historical perspective. Disability Studies Quarterly, 32(2).

Olusanya, B. O., \& Newton, V. E. (2007). Global burden of childhood hearing impairment and disease control priorities for developing countries. The Lancet, 369(9569), 1314-1317.
Olusanya, B. O., Ruben, R. J., \& Parving, A. (2006). Reducing the burden of communication disorders in the developing world: an opportunity for the millennium development project. JAMA, 296(4), 441444.

Padgett, D. (2008). Qualitative methods in social work research. Thousand Oaks: Sage.

Parker, G., Ali, S., Ringell, K., \& McKay, M. (2014). Bi-directional exchange: the cornerstone of globally focused social work. Global Social Welfare, 1(1), 1-8.

Reynolds, S. (2010). Disability culture in West Africa: qualitative research indicating barriers and progress in the greater Accra region of Ghana. Occupational Therapy International, 17(4), 198-207.

Scherzer, A. L., Chhagan, M., Kauchali, S., \& Susser, E. (2012). Global perspective on early diagnosis and intervention for children with developmental delays and disabilities. Developmental Medicine \& Child Neurology, 54(12), 1079-1084.

Siklos, S., \& Kerns, K. A. (2007). Assessing the diagnostic experiences of a small sample of parents of children with autism spectrum disorders. Research in Developmental Disabilities, 28(1), 9-22.

Strauss, A. L., \& Corbin, J. (1990). Basics of qualitative research (Vol. 15). Newbury Park: Sage.

Tuakli-Wosornu, Y. A., \& Haig, A. J. (2014). Implementing the world report on disability in West Africa: challenges and opportunities for Ghana. American Journal of Physical Medicine \& Rehabilitation, 93(1), S50-S57.

UNESCO International Bureau of Education (2006). Ghana early childhood care and education (ECCE) programmes. Retrieved from http://unesdoc.unesco.org/images/0014/001471/147192e.pdf on October 20, 2014.

United Nations Convention on the Rights of Persons with Disabilities (2006). Retrieved from http://www.un.org/disabilities/default.asp? id $=150$ on September 01, 2014.

Wetherby, A. M., Watt, N., Morgan, L., \& Shumway, S. (2007). Social communication profiles of children with autism spectrum disorders late in the second year of life. Journal of Autism and Developmental Disorders, 37(5), 960-975. 